\title{
Automated Traffic Signal Prediction from Surveillance Videos
}

\author{
Karthick.S \\ Student, \\ Department of Information \\ science and Technology, \\ College of Engineering, Guindy.
}

\author{
Deeban.B \\ Student, \\ Department of Information \\ science and Technology, \\ College of Engineering, Guindy.
}

\author{
S.Abirami \\ Assistant Professor, \\ Department of Information \\ science and Technology, \\ College of Engineering, Guindy.
}

\begin{abstract}
The traffic signals available in the present are based on the static feed of time without considering the actual available traffic. This leads to a situation where vehicles wait unnecessarily in one of the lanes while the traffic flow is not up to the considerable amount in the other lane. This paper provides a system to monitor the traffic flow automatically in traffic signals where video cameras are fixed. The time feed is made dynamic and automatic by processing the live traffic videos. The time for the signal is determined by two main factors: based on the density of the vehicles and on the number of vehicles in the lane. These inputs are given to a proposed algorithm which determines the optimal time period for the signal.
\end{abstract}

\section{General Terms}

Image processing, Traffic signal automation.

\section{Keywords}

Dynamic traffic signal prediction, density of vehicles, vehicular count.

\section{INTRODUCTION}

Vehicular detection techniques have been under research since 1970. As the need for reducing the installation and maintenance cost increased, the development of computer vision and widearea detection systems have evolved. Comparison between loop based and camera based techniques was funded by Minnesota Department of Transportation in 1990. The test proved the benefits of the camera-based techniques without even accounting other intangible benefits offered.The various other benefits provided via camera surveillance are as follows:

Knowledge:

Several patterns for traffic can be predicted. Moreover, the data for the laying roads, flyover construction is available.

Law:

Signal violation, traffic irregularities can be monitored and penalized.

\section{Safe Driving:}

Presence of surveillance cameras at traffic intersections cause drivers to drive with safe driving habits.

\subsection{Previous Work}

Vehicle detection techniques appear to be the weakest link in traffic surveillance and control system [1]. Closed loop, supersonic and radar method are already available. The drawback of these equipments is that they use "blind" type of detection technology and they cannot provide full traffic scene information in a human visual perspective manner. While detecting through surveillance cameras, videos provide more information than other sensors. Therefore, traffic surveillance systems based on videos provide reliable and robust traffic state information. As a result, monitoring by stationary video camera is one of the best techniques for traffic processing.

Various research projects to detect and track vehicles from stationary cameras have been carried out in the past decade. In traffic surveillance system, the evaluation of traffic conditions can be represented by the following parameters: traffic flow rate, average traffic speed, the length of queue, the number of vehicles and traffic density. Most of the proposed methods used to extract traffic information based on vehicle detection and tracking techniques. In these systems, robust and reliable vehicle detection and tracking is a critical step.

\subsection{Current Work}

In this paper, a novel method has been attempted to dynamically and automatically predict the traffic signal timer value. The live traffic video is given as input to the system. At first the background is extracted from the video by means of two frame differencing method. Next the density of the vehicles is calculated using the mean of the foreground estimated using two frame differencing method and background subtraction method. Once the foreground is estimated, the vehicular count is made by making use of the blob detection method. Finally, all of these parameters are given to the decision making algorithm. The most appropriate timer value for the signal is predicted. If the predicted value exceeds the maximum threshold, then maximum threshold is set as the timer value. This model has been tested in few traffic signals of Chennai city, as a part of the project funded by Centre of Technology Development and Transfer, Anna University Chennai. The remainder of the paper is organized as follows:

Section 2 gives system architecture design and explains the activities which are taking place in the system. Section 3 presents the various test cases and their results. Section 4 analyses the performance of the system. Section 5 concludes the work and gives suggestions for the future work. 


\section{SYSTEM ARCHITECTURE}

The overall architecture diagram of the system is shown in figure1.

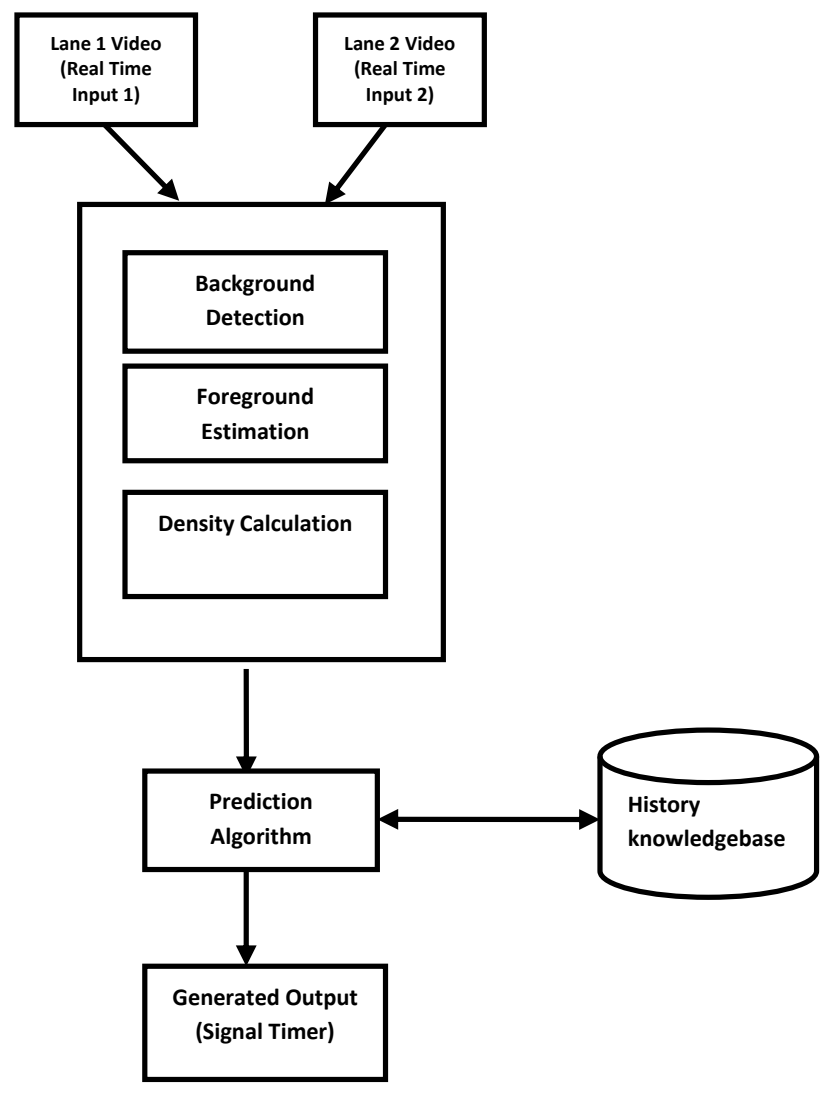

Fig 1: Traffic signal Prediction Architecture

\subsection{Traffic Video Inputs}

The input traffic video sequence comes from the cameras fixed at the signal junctions. Camera positioning and calibration techniques for integrating traffic surveillance video systems with machine-vision vehicle detection devices, has been obtained from [2]. Once the video input sequence is given to the system, the initial phases are the background extraction and foreground estimation processes.

\subsection{Background Detection}

Several background detection algorithms were developed over several years. Methods like Adaptive Background Modeling with Shadow Suppression [3], Background estimation for dynamic video scenes [4], An Enhanced Background Estimation Algorithm for Vehicle Detection in Urban Traffic Video [5] and advanced methods for detecting backgrounds are available. Since non calibrated camera is used, the accuracy of the estimated background is not of much importance. Hence in this system, we have decided to make use of the primitive method of background estimation based on the threshold value. We have chosen this so as to reduce the computational effort as the system is real time oriented. Figure 2 shows the sample estimated background of our system.

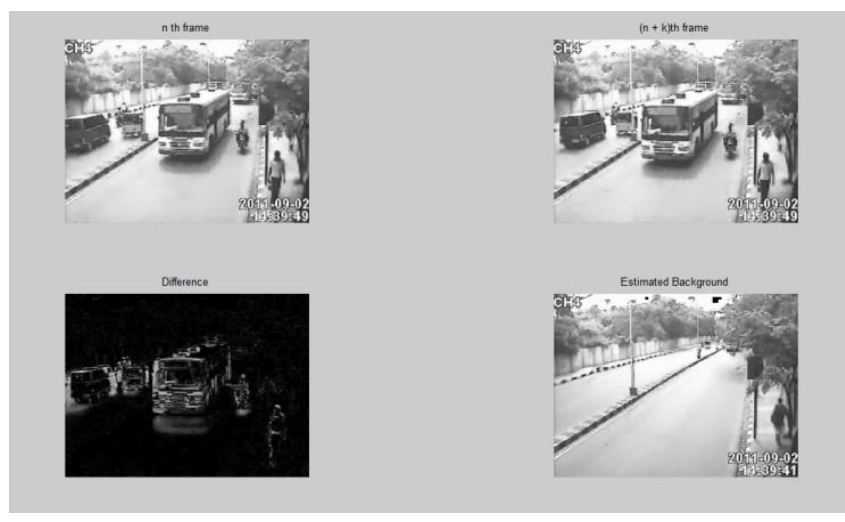

Figure 2 Clockwise top-left $a) n^{\text {th }}$ frame $\left.b\right)(n+k)^{\text {th }}$ frame c)Difference d)Estimated Background

\subsection{Foreground Estimation}

Once the background is detected, the estimation of the moving foreground becomes an easier task. In our system we made the following approaches for detecting the foreground.

We have obtained the background frame in the previous step of the system. We simply subtract the current frame with the estimated background frame. Stationary foreground detection has been done using background subtraction and temporal difference in video surveillance [6]. This gives the approximate foreground image. This is not so accurate due to noise or may be due to illumination changes or may also be due to errors in background frame estimated in the previous stage. Thus it will be inappropriate to carry out the error in the first phase till the end. This will lead to erroneous decisions. As a result we have also taken two frame differencing method while estimating the background.

In two-frame difference method, the difference between two frames is calculated. If the value is greater than the Threshold value, those pixels form the part of the foreground frame.

Thus the mean of the estimated foreground frames in both the methods has been chosen here. This gives the best approximation of the foreground image frame. The approximated image is morphologically opened and filled with white pixels. Figure 3 shows the estimated foreground image.

\subsection{Density Calculation}

Once the background and foreground images are estimated, next step is to calculate the density of the lane. The density of the lane is estimated based on the foreground image obtained in the last step of the process. 

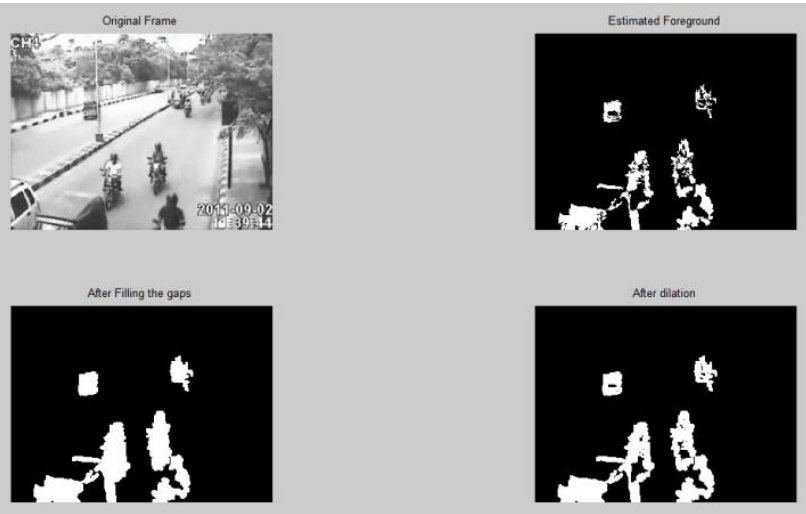

Fig 3: Clockwise Top-left a) Original frame b) Estimated Foreground c) After filling gaps d) After dilation

Density cannot be calculated precisely for the frame as a whole. There some difficulties in calculating the density of the lane which are as follows:

- The current frame consists of various sources of noises. Ex: Noise due to external factors, noise due to lighting conditions etc.

- The movement of camera may contribute to the noise.

- The other factors like pedestrians and animals may add to the density.

- Moreover the density of the vehicle in the farther region is difficult to calculate.

In order to overcome the above mentioned problems, we devise a three region approach. The frame under consideration is divided into three regions namely,

\section{Closest region, \\ 2. Center region, \\ 3. Point of infinity region.}

Closest region is the region which is close to the signal. It shows the traffic that already exists. Center region is the most important region. It shows the amount of traffic which will get accumulated in the next cycle. It helps us in determining the rate of growth in density. Finally, the Point of Infinity region is not of much importance. Vehicular count is done using the density of vehicles. The density is calculated in each of the regions separately. Figure 4 shows the splitting of frames into 3 regions.

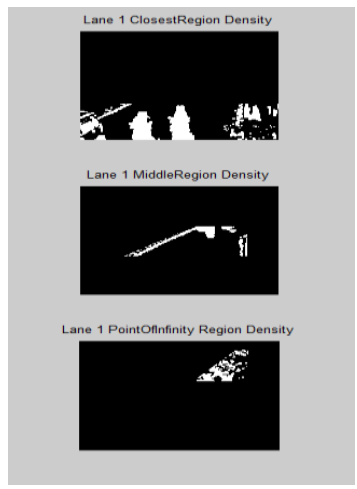

Fig 4: Splitting the frame into 3 regions

\subsection{Vehicular Count}

In order to make the system robust and stable, we need to consider another factor for predicting the traffic timer. Thus we predict the number of vehicles in the frame in region wise manner.

In order to detect the vehicles, we consider the foreground image obtained from the previous stage. The vehicles cannot be detected when the frame is considered as a whole. This is because the size of a vehicle, say a bike will be very small in the point of infinity region which keeps on increasing as it approaches the signal. It will be in the normal size in the closest region. Thus the approach used in density calculation is again based on zones.

The logically connected components are studied in each of the three regions separately. Separate thresholds are fixed (based on experimental values) for vehicle identification for the three regions. Suppose the logically connected region is greater than the threshold, then it is assumed to be a vehicle. The threshold is based on the smallest vehicle (bike in our example). If it is very large than the threshold then two cases are possible. The detected vehicle may be large (ex. Bus) or there is very little gap between the two vehicles which has lead to the large value that is occlusion between 2 vehicles. Thus, based on how much large the logically connected region is, than the threshold, the vehicle count is incremented accordingly.

The algorithm is applied to all the three regions respectively. This is given as input to the prediction algorithm as discussed subsequently. Similar works have been done in Automatic Vehicle Counting from Video for Traffic Flow Analysis [7], vehicle detection and counting from a video frame [8] and Moving Vehicle Detection for Automatic Traffic Monitoring [9]. Figure 5 shows the count of number of vehicles in the 3 regions.

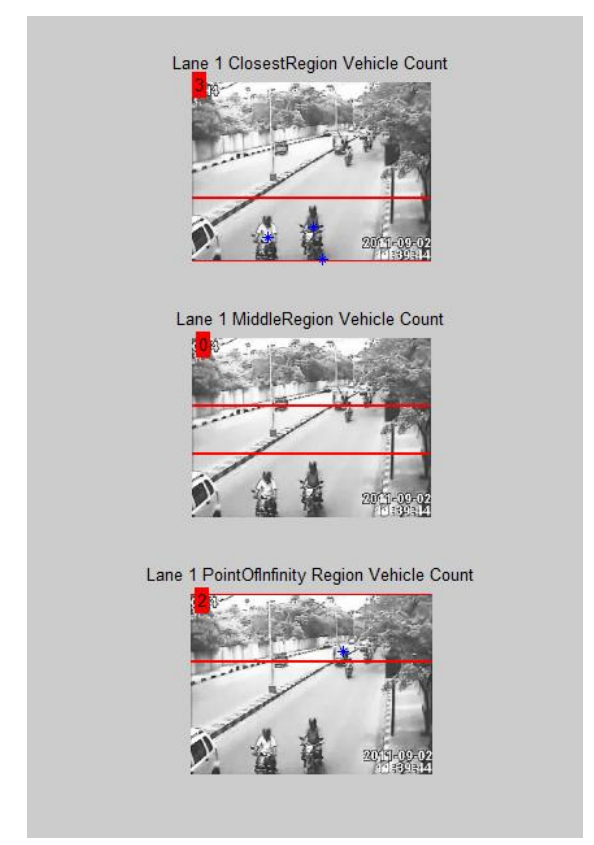

Fig 5: Counting the number of vehicles in the 3 regions. 


\subsection{Signal Prediction}

The density of the three regions and their corresponding vehicular count are obtained. This is now made use of, to predict the timer value for the next cycle. There are 9 cases with respect to the obtained density and vehicular count values. The algorithm is as follows:

Val will be the initial timer feed value. The timer feed value is modified based on various cases.

\section{Case 1: Consider Region 1}

Increase Val if the difference between the densities of the current and previous frames for region 1 is greater than the maximum threshold density value.

Increase Val if the difference between the count of the current and previous frames of region1 is greater than the maximum threshold count value.

\section{Case 2: Consider Region 2}

Similarly to case $1 \mathbf{V a l}$ is altered for the region 2 .

Case 3: Compare Region 1 and Region 2 of the same frame. Increase Val if the difference between the densities of the current frames of region 2 and 1 is greater than the minimum threshold density value.

Increase Val if the difference between the count of the current frames of region 1 and 2 is greater than the minimum threshold count value.

Case 4: Region 2 of previous frame and region 1 of current frame comparison

Increase Val if the between the count of the current frame's region 1 and previous frame's region 2 is greater then the maximum threshold count.

\section{Case 5: Check Region 3}

If Current frame Density greater than the Region 3 Threshold then increment the Val.

\section{Case 6: Look out for continuous increase or decrease}

If the Current Frame Density greater than the minimum threshold Density then there $s$ a buildup of traffic so pattern recognition variable is incremented, else the pattern recognition variable is decremented.

Pattern recognition variable is modified for all the frames which are processed.

Case 7: Consider the last frame before determining the Timer value

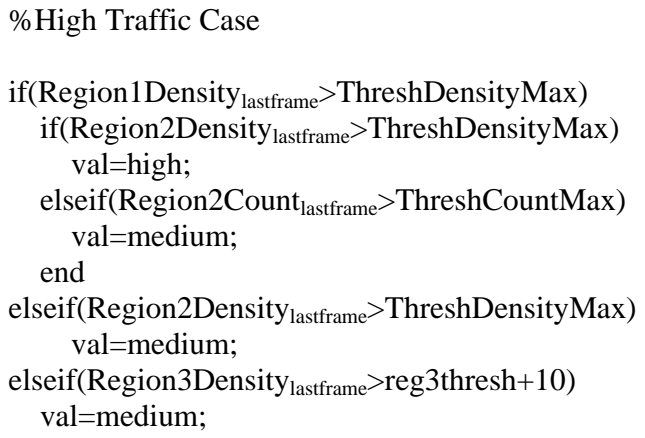

\%Low Traffic case
elseif(Region1Density lastrame $_{\text {ThreshDensityMin }}$

$\& \&$

Region1Count lastframe $<$ ThreshCountMin)

if(Region2Density lastframe $<$ ThreshDensityMin

Region2Count $_{\text {lastframe }}<$ ThreshCountMin)

if(Region3Density lastframe $_{\text {reg }}<$ thresh +10 ) val=low;

$$
\text { end }
$$

elseif(Region2Density lastframe $_{\text {ThreshDensityMax) }}$

$$
\text { val=val-7; }
$$$$
\text { end }
$$

end

\section{Case 8: SET the pattern}

If the pattern recognition variable at the end of processing is positive then the pattern is set to be increasing in nature, so maximum time feed value can be altered.

If the pattern recognition variable at the end of processing is negative then the pattern is set to be decreasing in nature, so minimum time feed value can be altered.

Case 9: Look at the history of values before deciding the timer value

if(ceil(cnt_avg) $<$ ThreshCountMin

den_avg $<$ ThreshDensityMin)

val=very low;

elseif(den_avg $>$ ThreshDensityMax

||cnt_avg>ThreshCountMax+10)

$$
\text { val=high; }
$$

end

\section{PERFORMANCE ANALYSIS \\ RESULTS}

The performance of the system can be measured by the accuracy with which the system predicts the time feed for the signal. Here the Precision has been calculated as

Precision $=$ Total no. of situations correctly predicted $/$ total no. of situations predicted.

There are three possibilities of the amount of traffic that could be found in the lanes

$$
\begin{aligned}
& \text { 1. High density. } \\
& \text { 2. Medium } \\
& \text { 3. Low density. }
\end{aligned}
$$

Considering the single lane alone, precision value depends on the accuracy with which the density is predicted and the vehicular count is taken. Table 1 and table 2 shows the vehicular count accuracy and density values obtained for high dense region.

\section{TABLE 1: VEHICULAR COUNT ACCURACY}

\begin{tabular}{|c|c|c|c|c|}
\hline & $\begin{array}{c}\text { Total no. of } \\
\text { Vehicles }\end{array}$ & $\begin{array}{c}\text { Small } \\
\text { (Two- } \\
\text { wheelers })\end{array}$ & $\begin{array}{c}\text { Medium } \\
\text { (Cars) }\end{array}$ & $\begin{array}{c}\text { Big } \\
\text { (Bus) }\end{array}$ \\
\hline Real & 16 & 12 & 3 & 1 \\
\hline Reported & 14 & 10 & 3 & 1 \\
\hline Accuracy & $88 \%$ & $84 \%$ & $100 \%$ & $100 \%$ \\
\hline
\end{tabular}


TABLE 2: DENSITY VALUE

\begin{tabular}{|c|c|}
\hline & Density Pixel count (in \%) \\
\hline Real & 75 \\
\hline Reported & 75 \\
\hline Accuracy & $100 \%$ \\
\hline
\end{tabular}

Note: There might be an increase in the pixel count density determined, than the original density because of the noise interferences.

Table 3 and table 4 shows the vehicular count accuracy and density values obtained for medium dense region.

TABLE 3: VEHICULAR COUNT ACCURACY

\begin{tabular}{|c|c|c|c|c|}
\hline & $\begin{array}{c}\text { Total no. of } \\
\text { Vehicles }\end{array}$ & $\begin{array}{c}\text { Small } \\
\text { (Two- } \\
\text { wheelers })\end{array}$ & $\begin{array}{c}\text { Medium } \\
\text { (Cars) }\end{array}$ & $\begin{array}{c}\text { Big } \\
\text { (Bus) }\end{array}$ \\
\hline Real & 9 & 5 & 3 & 1 \\
\hline Reported & 9 & 5 & 3 & 1 \\
\hline Accuracy & $100 \%$ & $100 \%$ & $100 \%$ & $100 \%$ \\
\hline
\end{tabular}

TABLE 4: DENSITY VALUE

\begin{tabular}{|l|l|}
\hline & Density Pixel count (in \%) \\
\hline Real & 40 \\
\hline Reported & 45 \\
\hline Accuracy & $89 \%$ \\
\hline
\end{tabular}

Table 5 and table 6 shows the vehicular count accuracy and density values obtained for less dense region.

TABLE 5: VEHICULAR COUNT ACCURACY

\begin{tabular}{|c|c|c|c|c|}
\hline & $\begin{array}{c}\text { Total no. of } \\
\text { Vehicles. }\end{array}$ & $\begin{array}{c}\text { Small } \\
\text { (Two- } \\
\text { wheelers })\end{array}$ & $\begin{array}{c}\text { Medium } \\
\text { (Cars) }\end{array}$ & $\begin{array}{c}\text { Big } \\
\text { (Bus) }\end{array}$ \\
\hline Real & 5 & 3 & 1 & 1 \\
\hline Reported & 5 & 3 & 1 & 1 \\
\hline Accuracy & $100 \%$ & $100 \%$ & $100 \%$ & $100 \%$ \\
\hline
\end{tabular}

TABLE 6: DENSITY VALUE

\begin{tabular}{|l|l|}
\hline & $\begin{array}{c}\text { Density Pixel count } \\
\text { (In \%) }\end{array}$ \\
\hline Real & 25 \\
\hline Reported & 30 \\
\hline Accuracy & $84 \%$ \\
\hline
\end{tabular}

Now with the count and the estimated densities as the input to the signal prediction algorithm, the time feed is predicted for signal updating.

Precision $=$ Total no. of situations correctly predicted $/$ Total no . of situations predicted.
TABLE 7: SIGNAL PREDICTION ACCURACY

\begin{tabular}{|l|l|l|l|}
\hline & Low (Lane2) & $\begin{array}{l}\text { Medium } \\
\text { (Lane2) }\end{array}$ & $\begin{array}{l}\text { High } \\
\text { (Lane2) }\end{array}$ \\
\hline Low(Lane1) & $100 \%$ & $95 \%$ & $90 \%$ \\
\hline Medium (Lane1) & $95 \%$ & $90 \%$ & $85 \%$ \\
\hline High(Lane1) & $90 \%$ & $85 \%$ & $80 \%$ \\
\hline
\end{tabular}

\section{CONCLUSIONS AND FUTURE WORK}

As a result, this system achieved $85 \%$ efficiency in predicting the signals. Prediction of signal for a single junction has been made. This work can be extended by taking the neighboring signals distributively into account and predicting the signal timing accordingly so that congestion can be avoided. Also, multiple actions can be performed from the video sequence in future to satisfy the following situations:

i. Video feeds can be shared with law enforcement agencies and fire departments during an incident.

ii. The video can also be used with license plate number recognition to find the vehicles which violate the traffic rules.

iii. Continuous monitoring to find vehicle trajectories which can be used for expansion of roads or building new intersections or flyovers.

iv. The system can also be extended to high efficient night surveillance.

v. System can be made intelligent so that it can sense emergency situations like ambulance and can change signals dynamically considering the situation.

\section{ACKNOWLEDGEMENTS}

This research is supported by the Centre for Technology Development and Transfer (CTDT), Anna University Chennai. The authors would like to thank the faculties of Department of Information Science and Technology, Anna University Chennai and the Department of Police (Traffic) Chennai, for providing us valuable support and guidance to complete this project.

\section{REFERENCES}

[1] J.M. Odobez and P. Bouthemy, "Robust Multi resolution Estimation of Parametric Motion Models", J. Visual Comm. and Image Representation, vol. 6, pp. 348-365, 1995

[2] Brian L. Smith, Ph.D., Assistant Professor, Department of Civil Engineering, University of Virginia , "Camera positioning and calibration techniques for integrating traffic surveillance video systems with machine-vision vehicle detection devices." , Virginia transportation research council.

[3] Hongzhe Han, Zhiliang Wang, Jiwei Liu, Zhengxi Li, Bin Li, and Zhongtao Han, "Adaptive Background Modeling with Shadow Suppression", Intelligent Transportation Systems, pp 720-724, IEEE 2003. 
[4] Sebastien Harasse, Laurent Bonnaud, Michel Desvignes. "Background estimation for dynamic video scenes", pp 3519-3524, IEEE 2006

[5] M. Vargas, Member, IEEE, S. L. Toral, F. Barrero, J.M Milla, "An Enhanced Background Estimation Algorithm for Vehicle Detection in Urban Traffic Video" Proceedings of the 11th International IEEE, Conference on Intelligent Transportation Systems, Beijing, China, pp. 784-790, October 2008.

[6] Álvaro Bayona, Juan C. SanMiguel, José M. Martínez, "Stationary foreground detection using background subtraction and temporal difference in video surveillance", 2010 IEEE 17th International Conference on Image Processing, Hong Kong, pp. 4657-4660, September 2010.
[7] Erhan Baş, A. Murat Tekalp, Fellow, IEEE, and F. Sibel Salman, "Automatic Vehicle Counting from Video for Traffic Flow Analysis", Proceedings of the 2007 IEEE Intelligent Vehicles Symposium Istanbul, Turkey, pp 392397,June 2007.

[8] Chomtip Pornpanomchali, Thitinut Liamsanguan, Vissakorn Vannakosit, "Vehicular detection and counting from a video frame", Proceedings of the 2008 International Conference on Wavelet Analysis and Pattern Recognition, Hong Kong, 30-31, pp. 356-361, August 2008.

[9] Jie Zhou, Senior Member, IEEE, Dashan Gao, and David Zhang, Senior Member, IEEE, "Moving Vehicle Detection for Automatic Traffic Monitoring" IEEE Transaction on vehicular technology, VOL. 56, NO. 1, pp 51-59, January 2007. 